\title{
Molecular Mechanisms and Drug Development in Aquaporin Water Channel Diseases: \\ Structure and Function of Aquaporins
}

\author{
Masato Yasui ${ }^{1, *}$ \\ ${ }^{I}$ Departments of Pediatrics and Biological Chemistry, Johns Hopkins University School of Medicine, \\ Baltimore, MD 21205, USA
}

Received September 29, 2004; Accepted October 13, 2004

\begin{abstract}
The discovery of the water channel aquaporin has greatly expanded our understanding of the regulation of the water permeability of biological membranes. The atomic structure of aquaporin-1 (AQP1) demonstrated how aquaporin is freely permeated by water but not protons and provided marked insight into several human disorders. Eleven mammalian aquaporins have been identified, each with a distinct distribution, and these are selectively permeated by water or water plus glycerol. Aquaporins are suspected in numerous pathological conditions involving fluid transport such as brain edema. Knowledge of aquaporin structure may provide insight into the development of new therapeutics through appropriate drug design.
\end{abstract}

Keywords: aquaporin-1, permeability, water channel, structure and function, human disease

\section{Introduction}

Water constitutes roughly $70 \%$ of our body mass; the appropriate distribution of water is required to maintain orderly fluid balance within different anatomic compartments. The proper regulation of water permeability of cell membranes is therefore a fundamental requirement for our life. In contrast to the classic view of simple diffusion through the lipid bilayer of cell membranes, biophysical and physiological studies over several decades have predicted the existence of a membrane-channel-protein-mediated water movement in certain membranes (1). The molecular identification of such a channel protein became apparent during the early 1990s with the discovery of the first water channel protein, CHIP 28 (now called aquaporin-1 or AQP1) (Fig. 1). Discovery of the aquaporin family of waterchannel proteins has provided new insights into the molecular mechanisms of trans-membrane water movement (2). At least 11 aquaporins have been identified in mammals (3). It is becoming apparent that aquaporin biology will prove relevant to the pathophysiology and perhaps even therapy to treat a wide range of conditions.

*Corresponding author. E-mail: myasui@jhmi.edu

\section{The atomic structure}

The primary sequence of the AQP1 cDNA revealed two tandem repeats each containing three bilayerspaning $\alpha$-helices. The loops connecting the second and third membrane-spanning $\alpha$-helix in each repeat contained the signature motif, asparagines-prolinealanine (NPA). Based on biochemical and site-directed mutagenesis studies, an 'hourglass model' was proposed: a pseudo two-fold symmetrical structure with the six bilayer-spanning $\alpha$-helices surrounding the

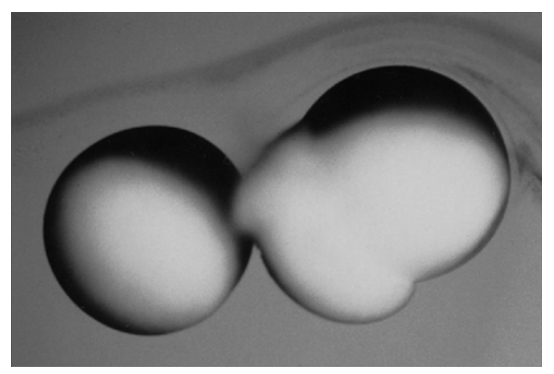

Fig. 1. Functional expression of AQP1 in Xenopus laevis oocytes. An oocyte injected with AQP1 cRNA (right) has swollen and exploded in hypotonic buffer. In contrast, a control oocyte injected with water fails to swell (left). Reprinted from Ref. 2 with permission (Copyright 1992 AAAS). 
aqueous pore formed from the two NPA-containing loops that enter the bilayer from the opposite surfaces and overlap at the junction of the two NPA motifs (4). Several years later, reconstitution of purified AQP1 from human erythrocytes into proteoliposomes allowed the establishment of its structure. The membrane crystals were analyzed by atomic force microscopy as well as electron microscopy $(5,6)$. A series of studies led to the same general conclusion supporting the 'hourglass model' for the AQP1 monomer (4). Note that AQP1 exists as a tetramer with each subunit containing its own aqueous pore.

The structure of AQP1 provides a uniquely selective mechanism for free permeation by water through a channel pore and a mechanism for proton blockage (7). The ability to block proton transport clarifies how the kidneys can reabsorb hundreds of liters of water from glomerular filtrate each day while excreting acid. The first barrier to proton permeation is located at the narrowest of the extracellular mouth of the pore. The residue at the constriction Arg-195 is nearly perfectly conserved and bears a strong positive charge to repel proton. The second barrier to proton permeation is at the midway of the pore with strong dipole, formed by the two short pore helices containing the NPA motif. Both structural studies and molecular dynamics simulations have proven that these partial charges at the center of AQP1 and Asn residues in the NPA motif reorient water molecules passing through the channel (8). This water dipole reorientation disrupts hydrogen-bonding interactions between this water molecule and those both above and beneath it, eliminating the possibility of proton conductance (Fig. 2).

\section{Variety of aquaporin family}

Since AQP1 was identified as a molecular water channel, hundreds of homologous proteins have been recognized in plants, microbes, invertebrates, and vertebrates. Eleven mammalian aquaporins have now been identified and at least partially characterized. These conform to two subsets of proteins - those selectively permeated by water (classic aquaporins) and those permeated by water and glycerol (aquaglyceroporins) (Fig. 3). The atomic structure of the E. coli aquaglycroporin GlpF has been solved, and transport of glycerol through the channel has been simulated by molecular dynamics (9). These data indicate that GlpF, with a glycine residue in the position analogous to His-180, has a pore diameter approximately $1 \AA$ wider than that of AQP1, permitting lengthwise passage of glycerol (Fig. 3).

The functional repertoire of aquaporins was expanded

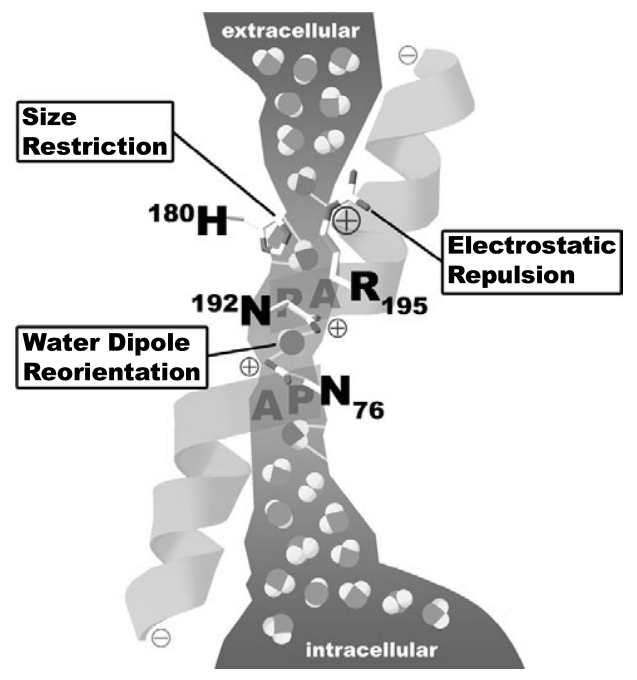

Fig. 2. Schematic architecture of the channel pore within AQP1 monomer. Three features of the channel specify selectivity for water: 1) Size restriction. 2) Electrostatic repulsion. 3) Water dipole reorientation. See text for detailed explanations. Reprinted from Ref. 7 with permission.

by discovery of AQP6 (10). Although AQP6 belongs to the family of classic aquaporins based on the homology in primary amino acid sequence, AQP6 is not a simple water channel. Very surprisingly, AQP6 behaves as a $\mathrm{pH}$-regulated anion channel with greatest selectivity for nitrate (Fig. 4) (11). This unique functional property of AQP6 predicts that AQP6 might have a distinct pore structure. We have recently identified an amino-acid residue in AQP6 critical for its anion permeability by site-directed mutagenesis. Interestingly, the mutant AQP6 protein turns out to be a functional water channel without any ion permeability (manuscript in preparation). We expect that a structural comparison between the wild-type AQP6 and the mutant AQP6 will provide clues about the general mechanism of ion blocking for aquaporins. AQP6 is exclusively expressed in acidsecreting $\alpha$-intercalated cells of the renal collecting duct where the protein is restricted to intercellular sites co-localized alongside H-ATPase, suggesting that AQP6 may participate in acid-base regulation (12). Very recently we also found AQP6 in the cerebellum as well as several other tissues (unpublished). The phenotypic analysis of AQP6-null mice is being used to identify the physiological relevance of this aquaporin.

\section{Aquaporin and human clinical disorders}

The atomic structure of AQP1 provides not only detailed understanding into molecular mechanisms for protein dysfunction but marked insight into several human disease states (7). 


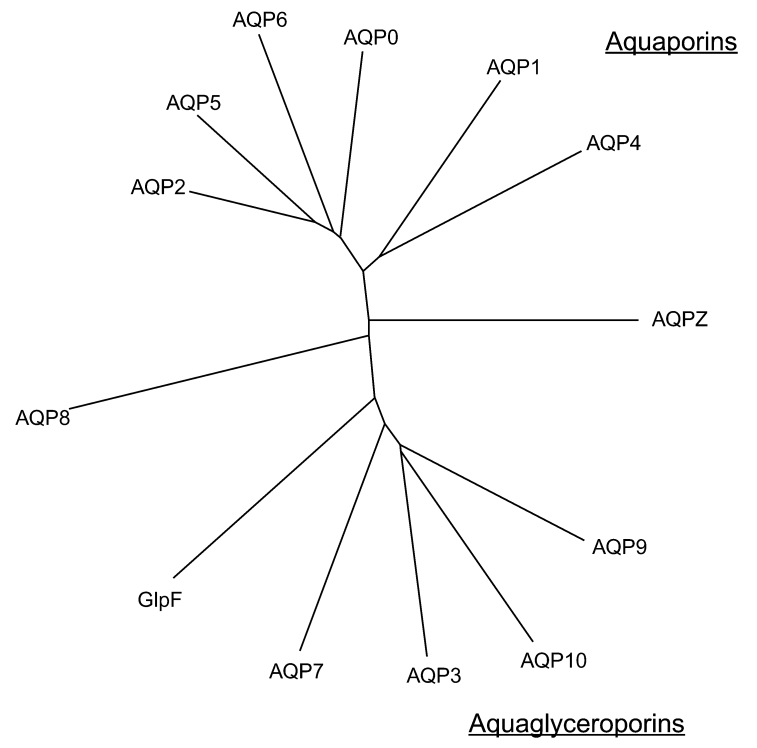

0.1
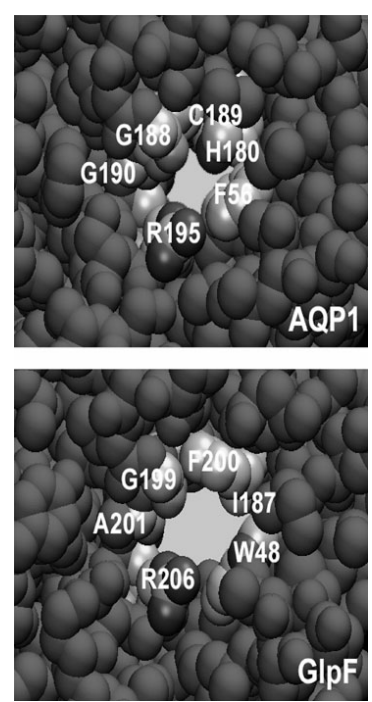

Fig. 3. Human aquaporin gene family with E. coli homologs. Water permeable (aquaporins) and glycerol permeable (aquaglyceroporins) family members are shown (left). Space-filling models of AQP1 and GlpF reveal the difference in pore size (right).
A

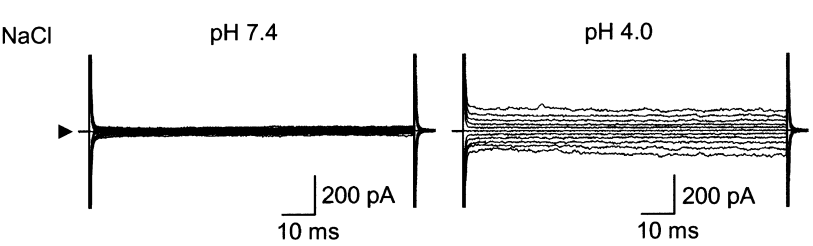

C

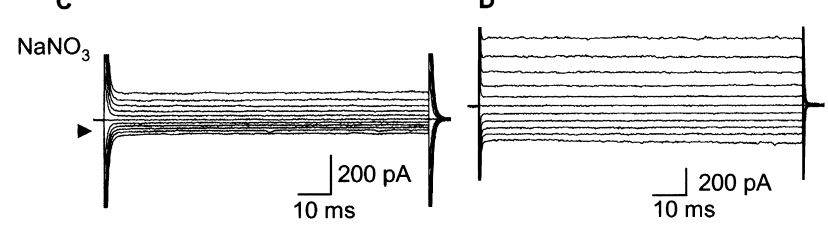

$E$

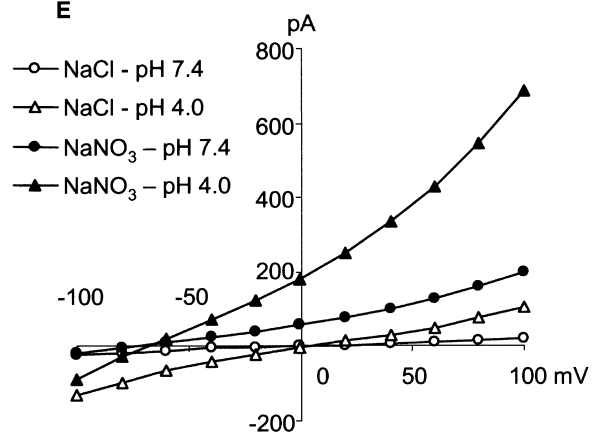

Fig. 4. pH-regulated anion permeability of AQP6. Current responses induced by the replacement of $\mathrm{Cl}^{-}$with $\mathrm{NO}_{3}^{-}$in GFPAQP6-expressing cells. Representative currents from the same GFP-AQP6-expressing cell in $\mathrm{NaCl}$ solution $(\mathrm{A}$ and $\mathrm{B})$ and $\mathrm{NaNO}_{3}-$ solution $(\mathrm{C}$ and $\mathrm{D})$ at $\mathrm{pH} 7.4$ (left traces) and $\mathrm{pH} 4$ (right traces) are shown. The corresponding I-V relationships are depicted in E. Reprinted from Ref. 11 with permission.
$\mathrm{AQP0}$ is the major intrinsic protein of lens fiber cells. Inherited defects in the gene encoding AQP0 have been identified in two large kindreds from the United Kingdom with dominantly inherited cataracts $(13,14)$. The affected family members carry single amino acid substitutions, Glu-134-Gly or Thr-138-Arg. Glu-134 is conserved among all aquaporins; the atomic structure of AQP1 indicates that this residue lies near the highly conserved residue Arg-187 and may restrict the orientation of loop E within the six transmembrane domains. The Thr-138-Arg mutation may perturb the proper orientation of Glu-134 by introducing an adjacent positive charge. The occurrence of early onset cataracts caused by major defects in the AQP0 structure strongly suggests that less severe defects will be found in some patients with typical, late-onset cataracts common in aged people.

The individuals lacking AQP1 protein were identified because they lack the Co blood group antigen (15). Surprisingly, the AQP1-null individuals led normal lives and were entirely unaware of any physical limitations. Careful clinical analyses of the AQP1-null individuals, however, revealed mild renal concentration defects, which could become significant if the individuals should be deprived of water (16).

AQP2 is predominantly localized in the principal cells on the renal collecting ducts where regulated water reabsorption takes place in response to antidiuretic hormone, vasopressin (17). This suggests that AQP2 may be involved in some types of nephrogenic diabetes inspidus (NDI): a majority of congenital NDI is due to mutations in the gene for the vasopressin $V_{2}$ receptor 
located in X g 28. Patients with recessively inherited NDI have been identified as having mutations in the AQP2 gene, affecting the formation of the aqueous pore of the protein; one mutation was found in Arg-187, which disrupts the normal folding of the narrowest constriction region in the aqueous pore (18). Subsequently, a family with dominantly inherited NDI was identified, and the site of mutation was found in the carboxy-terminus near the PKA phosphorylation site that regulates exocytosis (19).

\section{Clinical pharmacology}

Aquaporins are suspected in numerous disorders involving fluid transport such as brain edema, lung edema, NDI, dry eye, and congestive heart failure (20). Recent advances in determining the structures of aquaporins at the atomic level have explained molecular mechanisms in defects of the proteins and expanded our understanding of aquaporin-related diseases (21). Mercury has been known to inhibit water transport through aquaporins by interacting with the Cys-189 residue, predicted to reside within the constriction of the pore (22). However, we need to develop more specific agents that will serve to inhibit or activate the aquaporins. Knowledge about regulatory mechanisms of aquaporin may facilitate appropriate drug design.

\section{Acknowledgments}

The author wishes to thank David Kozono for helpful discussions and for assistance with figures. This work was supported by grants from the National Institutes of Health, the American Heart Association, and the S\&R Foundation.

\section{References}

1 Finkelstein A. The red cell membrane. In: Water movement through lipid bilayer, pores, and plasma membranes. New York: Wiley; 1987. p. 166-184.

2 Preston GM, Carroll TP, Guggino WB, Agre P. Appearance of water channels in Xenopus oocytes expressing red cell CHIP28 protein. Science. 1992;256:385-387.

3 Agre P, King LS, Yasui M, Guggino WB, Otterson OP, Fujiyoshi $\mathrm{Y}$, et al. Aquaporin water channels - from atomic structure to clinical medicine (Topical Review). J Physiol (Lond). 2002;542:3-16.

4 Jung JS, Preston GM, Smith BL, Guggino WB, Agre P. Molecular structure of the water channel through aquaporin CHIP. The hourglass model. J Biol Chem. 1994;269:14648-14654.

5 Murata K, Mitsuoka K, Hirai T, Walz T, Agre P, Heymann JB, et al. Structural determinants of water permeation through aquaporin-1. Nature. 2000;407:599-605.
6 Sui H, Han BG, Lee JK, Walian P, Jap BK. Structural basis of water-specific transport through the AQP1 water channel. Nature. 2001;414:872-878.

7 Kozono D, Yasui M, King LS, Agre P. Aquaporin water channels: atomic structure and molecular dynamics meet clinical medicine (review). J Clin Invest. 2002;109:1395-1399.

8 Tajkhorshid E, Nollert P, Jensen MO, Miercke LJ, O'Connell J, Stroud RM, et al. Control of the selectivity of the aquaporin water channel family by global orientational tuning. Science. 2002;296:525-530.

9 Fu D, Libson A, Miercke LJ, Weitzman C, Nollert K, Kruncinski J, et al. Structure of a glycerol-conducting channel and the basis for its selectivity. Science. 2000;290:481-486.

10 Yasui M, Hazama A, Kwon T-H, Nielsen S, Guggino WB, Agre P. Rapid gating and anion permeability of an intracellular aquaporin. Nature. 1999;402:184-187.

11 Ikeda M, Beitz E, Kozono D, Guggino WB, Agre P, Yasui M. Characterization of aquaporin- 6 as a nitrate channel in mammalian cells: requirement of pore-lining residue threonine-63. J Biol Chem. 2002;277:39873-39879.

12 Yasui M, Kwon T-H, Knepper MA, Nielsen S, Agre P. Aquaporin-6: an intracellular vesicle water channel protein in renal epithelia. Proc Natl Acad Sci USA. 1999;96:5808-5813.

13 Berry V, Francis P, Kaushal S, Moore A, Bhattacharya S. Missense mutations in MIP underlie autosomal dominant polymorphic and lamellar cataracts linked to $12 \mathrm{q}$. Nat Genet. 2000;25:15-17.

14 Francis P, Chung JJ, Yasui M, Berry V, Moore A, Wyatt MK, et al. Functional impairment of lens aquaporin in two families with dominantly inherited cataracts. Hum Mol Genet. 2000;9: 2329-2334.

15 Preston GM, Smith BL, Zeidel ML, Moulds JJ, Agre P. Mutations in aquaporin-1 in phenotypically normal humans without functional CHIP water channels. Science. 1994;265: $1585-1587$.

16 King LS, Choi M, Fernandez PC, Cartron JP, Agre P. Defective urinary-concentrating ability due to a complete deficiency of aquaporin-1. N Engl J Med. 2001;345:175-179.

17 Nielsen S, Chou Cl, Marples D, Christensen EI, Kishore BK, Knepper MA. Vasopressin increases water permeability of kidney collecting duct by inducing translocation of aquaporinCD water channels to plasma membrane. Proc Natl Acad Sci USA. 1995;92:1013-1017.

18 Deen PMT, Verdijk MAJ, Knoers NVAM, Wieringa B, Monnens LA, van Os $\mathrm{CH}$, et al. Requirement of human renal water channel aquaporin-2 for vasopressin-dependent concentration of urine. Science. 1994;264:92-95.

19 Nielsen S, Frokier J, Marples D, Kwon T-H, Agre P, Knepper MA. Aquaporins in the kidney: from molecules to medicine. Physiol Rev. 2002;82:205-244.

20 Manley GT, Fujimura M, Ma T, Noshita N, Filiz F, Bollen AW, et al. Aquaporin-4 deletion in mice reduces brain edema after acute water intoxication and ischemic stroke. Nat Med. 2000;6:159-163.

21 King LS, Yasui M, Agre P. Aquaporins in health and disease (review). Mol Med Today. 2000;6:60-65.

22 Preston GM, Jung JS, Guggino WB, Agre P. The mercury-sensitive residue at cysteine 189 in the CHIP28 water channel. J Biol Chem. 1993;268:17-20. 